\title{
Anti-Angiogenesis Therapy of Cancer Cells using ${ }^{153} \mathrm{Sm}-$ Bevasesomab
}

\author{
Kamal Yavari $\mathrm{a}^{*}$ \\ ${ }^{a}$ Nuclear Sciences and Technology Research Institute, Tehran, Iran
}

\begin{abstract}
Purpose: Angiogenesis is essential for tumor growth or metastasis. Avastin is a monoclonal antibody that is used in treating angiogenesis. We labelled this monoclonal antibody with samarium153 and performed in vitro quality control tests as a first step in the production of a new radiopharmaceutical.

Material and Methods: For a successful radiolabeling, we chose DOTA-NHS as the bifunctional chelating agent and optimized radiolabeling condition with modifications of the factors such as reaction time and molar ratio which are known to be very critical in radiolabeling. The efficiency and in vitro stability of antibody labelling were determined using thin layer chromatography. The integrity of the radiolabeled antibody was checked by SDS-PAGE. Biodistribution study of 153Sm-DOTA -avastin was carried out in BALB/c mice at 2, 24, 48 and 72 hours after injection. Immunoreactivity and toxicity of the complex were tested on colon cancer cell line by MTT.

Results: The efficiency of antibody labelling was more than $99 \%$. The in vitro stability of the labelled product in human serum after $120 \mathrm{~h}$ was $78 \pm 2 \%$. There was no fragmentation in the labelled antibody during SDS-PAGE protocol. The highest of \%ID/g was observed in the blood, liver, lungs and spleen. The immunoreactivity of the complex was $89 \pm 1.4 \%$. At a concentration of $1 \mathrm{nM}$, the complex killed $70 \pm 3 \%$ of SW480 cells. At $1.9 \mathrm{nM}, 90 \pm 5 \%$ of the cells were killed.

Discussion: The monoclonal antibody avastin against angiogenesis was effectively radiolabeled with $153 \mathrm{Sm}$. The results showed that the new complex could be considered a promising tracer for noninvasive delineation of angiogenesis.
\end{abstract}

Keywords:

Angiogenesis;

Bevasezomab;

${ }^{153} \mathrm{Sm}$;

DOTA-NHS;

Cancer Cells;

Proliferation;

Radioimmonoaffinity.

\section{Article History:}

Received: 28 January 2018

Accepted: 16 May 2018

\section{1- Introduction}

Tumors require angiogenesis in order to grow beyond the size of 1-2 $\mathrm{mm}$ in diameter, a condition in which nutrients and oxygen are not adequately supplied via passive diffusion through the existing blood vessels [1]. Furthermore, the metastatic property of tumors strongly correlates with tumor angiogenesis [2, 3]. In Particular, the balance between various activators and inhibitors is closely associated with overexpression of vascular endothelial growth factor (VEGF) and its receptors serves to regulate angiogenesis. It is well known that VEGF plays a pivotal role in angiogenesis proliferation and migration of endothelial cells. Newdayes, great interest lies in angiogenesis and in its potential clinical implications in cancer, and vascular targeting a promising strategy for cancer imaging and therapy. Numerous compounds possessing an antiangiogenic profile have been investigated [4-10]. Among potential therapeutic strategies to induce tumor regression by blocking tumor blood supply, an intriguing approach relies on the selective targeting of cell surface molecules over-expressed on endothelial cells of the tumor-associated blood vessels.

Radiolabeled monoclonal antibodies have been developed for both the diagnosis and treatment of tumors in targeted radiotherapy. In targeted radiotherapy, a $\beta$ - and/or $\gamma$-emitter radioisotope is coupled to at argeting agent that has several advantages over external beam radiotherapy, including the possibility of selectively delivering higher doses to the tumor and treating multiple metastasizes [10]. In addition, a radiolabeled targeting agent has two major advantages over the application of targeting agent alone. First, radionuclide can irradiate not only the specifically targeted tumor cells, but also the surrounding area, including tumor cells regardless of the target antigen (cross-fire phenomenon). Second,

\footnotetext{
* CONTACT: kyavari@aeoi.org.ir

DOI: http://dx.doi.org/10.28991/esj-2018-01136

(C) This is an open access article under the CC-BY license (https://creativecommons.org/licenses/by/4.0/).
} 
cytotoxicity of the radionuclide is less dependent than chemotherapeutic agents on cellular expression of discrete biochemical targets. In particular, suitable gamma energy enables us to track targeting agent both in vitro and in vivo $[11,12]$.

An ideal therapeutic radionuclide would be a $\beta$-emitter with suitable gamma energy enabling a simultaneous track of the labeled compound. ${ }^{153} \mathrm{Sm}$ is an ideal radionuclide for the imaging and therapy of tumors due to its favorable decay characteristics. ${ }^{153} \mathrm{Sm}$ is a radiolanthanide with a $\beta$-emission similar to ${ }^{131} \mathrm{I}$ but with two advantages over it. The main $\gamma$ photons of ${ }^{153} \mathrm{Sm}$ ( $208 \mathrm{KeV}, 11 \%$ abundance) are more suitable for imaging with a gamma camera than those of ${ }^{131} \mathrm{I}$ (364 KeV, 82\% abundance) without additional radiation dose that would comprise normal tissues. Furthermore ${ }^{153} \mathrm{Sm}$ does not need special design of residualization. Due to its physical characteristics, ${ }^{153} \mathrm{Sm}$ is being increasingly used in therapeutic research studies $[15,16]$.

In this study, we describe the optimal conditions for labeling of anti-VEGF mAb with ${ }^{153} \mathrm{Sm}$ using DOTA-NHS and investigate its in vitro and in vivo behaviors. We expect that this optimal procedure for radiolabeling of biomolecule is applicable to develop the novel radiolabeled peptides or angiogenesis inhibitors for targeted therapy.

\section{2- Materials and Methods}

\section{2-1- Materials}

Bevasezomab antibody purchased from Merck, Lotus and Samarium oxides were purchased from Trace.The DOTANHS Shotut was purchased from Macrocyclic Company Chlorideic Acid, Nitric Acid, Methanol, Ethanol, NaCl, $\mathrm{NH} 3 \mathrm{OH}$, Chloroform, DMSO, Acetic Acid, EDTA, Ammonium Acetate, Sodium Acetate and PBS from Mercury were purchased. Copper sulfate, Na2CO3, Na2HCO3, MTT, Trypsin, Xylasin and Ketamin, (Arsenazo (III and Cu (III) from Sigma. Sephodox G25 and G50 purchased from Pharmacy. SW480 cell lines and Balb /C mice purchased from the Institute Pasteur of Iran.

\section{2-2- Preparation of Conjugated Avastin}

DOTA-NHS (at a molar ratio of 50) was dissolved in $0.5 \mathrm{ml}$ bicarbonate buffer. Avastin solution $((5 \mathrm{mg}, 0.5 \mathrm{ml}))$ in a bicarbonate buffer was added slowly to a DOTA-NHS solution and slowly mixed at room temperature for 24 hours. The conjugation reaction was completed by adding $0.25 \mathrm{M}$ ammonium acetate, $\mathrm{pH}$ 5.5. The conjugate was then washed with the same buffer in an ultrafilter column. Filtration and washing were continued until the optical density of the filtered solution was close to zero while the filtrate was monitored by biophotometer at $280 \mathrm{~nm}$ and immunoconjugate was storaged at $4^{\circ} \mathrm{C}$.

\section{2-3- Determine the Mean Molar Ratio of Chelator to Antibody}

A spectrophotometric method was used to determine the number of chelating groups for each antibody. titration of the $\mathrm{Cu}$ (II)-Arsenazo (III) complex according to the method described by Pippin et al [17].

\section{2-4- Radiolabeling of Avastin}

In two separate glass vials, $3.7 \mathrm{MBq} / \mathrm{ml}$ of ${ }^{153} \mathrm{Sm}$ in a volume of 10 to $20 \mu \mathrm{lin} 0.2 \mu \mathrm{m} \mathrm{HCl}$ was added to $0.5 \mathrm{ml}$ of ammonium acetate buffer, then the conjugated avastin-NHS-DOTA was added and mixed slowly in $43^{\circ} \mathrm{C}$ for 1 hour. The avastin-DOTA- ${ }^{153} \mathrm{Sm}$ complex was purified by PD-10 desalting Columns contain Sephadex G-25 resin.

\section{2-5- Qualitative Control Tests}

\section{2-5-1- Radiochemical Purity}

The labeling efficiency was evaluated using thin layer chromatography (TLC) utilizing WattMan No. 1 tapes and 10mM EDTA: (v/v) as solvent.

\section{2-5-2- The Stability of Bevasezomab Labelledwith ${ }^{153} \mathrm{Sm}$}

The samples contain $1 \mathrm{ml}$ of human serum were prepared. An aliquot of $25 \mu 1$ of the ${ }^{153} \mathrm{Sm}$-DOTA-avastin complex

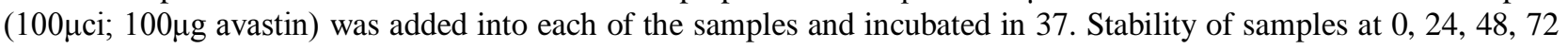
and 96 hour of incubation was determined using TLC. In order to verify the stability of the complex in various conditions including $4^{\circ} \mathrm{C}$ temperature, room temperature and phosphate buffer, a method similar to that of the serum stability was used.

\section{2-5-3- Integrity of Radioimonoconjugate}

The probability of occurrence of structural changes in avastin due to labeling with samarium radioisotope, was investigated using SDS-PAGE technique. 


\section{2-5-4- Immunoreactive Determination}

By doing this, we will make sure of any changes in the likelihood of antibody binding to the antigen. during the labeling process. Also, we determine the Antibody-Antigen binding feature. Cell binding was assessed by according to the method of Lindmo.

\section{2-5-5- Cell Growth Assay Using MTT}

The basis of this experiment is the formation of a colored complex of living cells (due to mitochondrial dehydrogenase enzyme) with MTT. For performing the experiment, $0.4 \times 10^{4}$ cells/well in 96-well plates were seeded to each 96-well plates containing $200 \mu \mathrm{l}$ of the culture medium. On the next day, the complex and controls treatment was performed according to the protocol. Treated and control cells were incubated in $5 \% \mathrm{CO} 2$ at $37^{\circ} \mathrm{C}$ for 4 days and MTT assay was done in every day. For MTT assay, $10 \mu 1$ of solution of MTT ( $5 \mathrm{mg} / \mathrm{ml}$ of PBS) was added to each well and incubated for $4 \mathrm{~h}$ at $37^{\circ} \mathrm{C}$. The plate was then removed from the incubator and the cells medium was removed. The Formosan crystals were dissolved in the wells by adding $200 \mu$ l of DMSO (dimethyl sulfoxide, sigma) and incobated for 15 minutes. The created color change was read at $570 \mathrm{~nm}$. To evaluate the cytotoxic effects of avastin-DOTA- ${ }^{153} \mathrm{Sm}$, four groups of cells were considered for each assay: group1, which did not receive any treatment; group 2 were that received free ${ }^{153} \mathrm{Sm}$ isotope; group3, which treated only with avastin and group4 of cells that treated with avastin-DOTA${ }^{153} \mathrm{Sm}$ complex.

\section{2-5-6- Stability and Biodistribution Studies of Avastin-DOTA-153Sm in Normal Mice}

In order to evaluate the normal distribution of the product, a volume $(0.1 \mathrm{~mL})$ of final ${ }^{153} \mathrm{Sm}$-DOTA-avastin or ${ }^{153} \mathrm{SmCl} 3$ solution containing $3.7 \mathrm{MBq}{ }^{153} \mathrm{Sm}$ was injected into a series of normal rats through a tail vein. The mice were anesthetized at 2, 24, 48, and 72 hours after treatment and blood samples were taken. Then, different tissues were separated and the radioactivity percentage of injected per gram of tissue (\%ID / g) was measured using HPGe system.

\section{3- Results and Discussion}

\section{3-1- Average Number of Chelates per Antibody}

On an average, 1.1-1.7 DOTA analogues were conjugated to avastin and the conjugation reactions took place at 20:1 for NHS-DOTA to avastin.

\section{3-2- Radiochemical Purity}

The relative counts recorded from the different segments of the chromatographic papers against the distance from the bottom of the papers are shown in Fig. 1 the highest activity is seen in the first segments showing high radiochemical purity. As can be seen, the radiochemical purity of the complex was $99 \pm 0.9 \%$ at $1 \mathrm{~h}$ and $\mathrm{pH}$ of 5.5 (Figure 1).

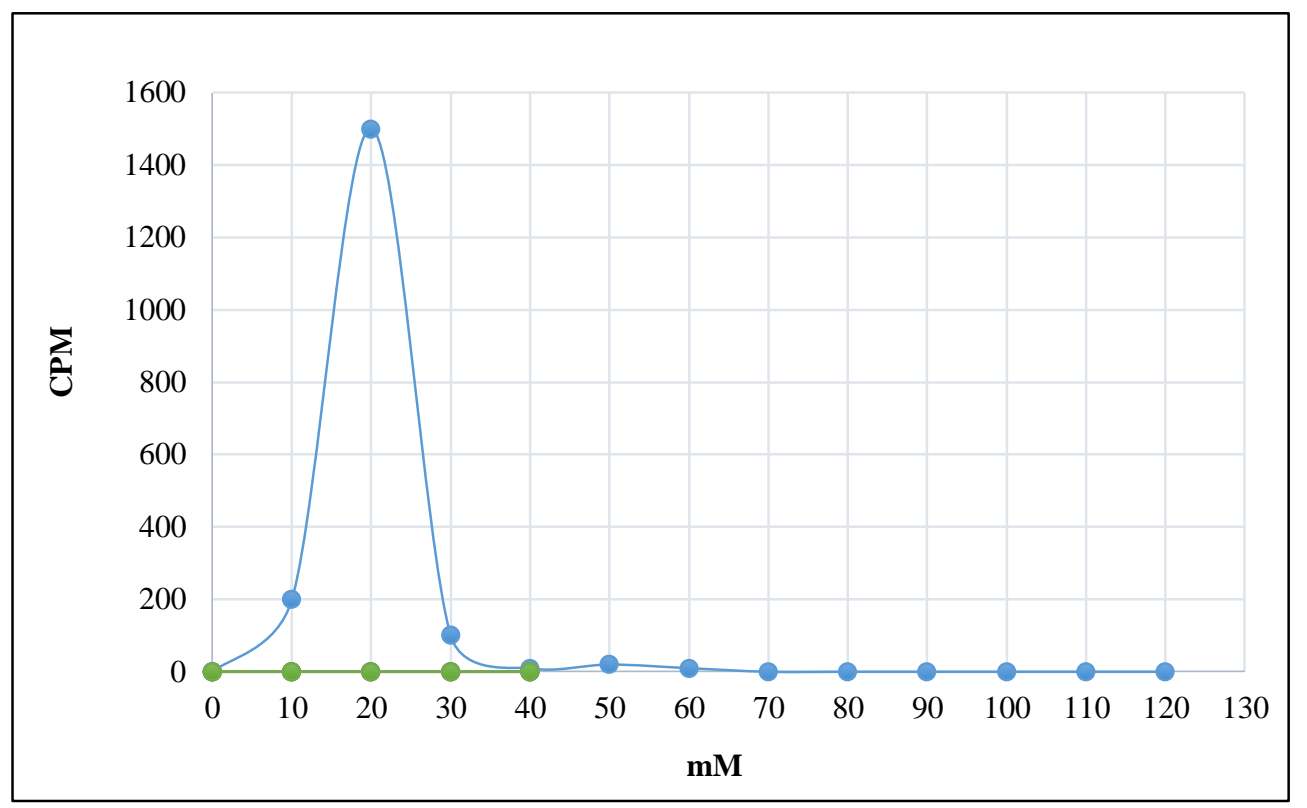

Figure 1. Radiochemical purity of labelling using the relative counts recorded from different segments of the chromatography paper against the distance from the bottom of the paper. 


\section{3-3- The Stability of Complex in Various Condition}

The stability of labelled antibody was evaluated by instant thin layer chromatography at $4^{\circ} \mathrm{C}$, room temperature, in the phosphate buffer and incubation in human plasma at $37^{\circ} \mathrm{C}$ for different times. Fig2 shows that ${ }^{153} \mathrm{Sm}$-DOTA-avastin remains stable at these temperatures and the radiochemical purity were higher than $96 \%$ for more than 120 hours of storage. Also the complex showed greater stability up to $120 \mathrm{~h}$ in human plasma (78 $\pm 2 \%$ ) (Figure 2).

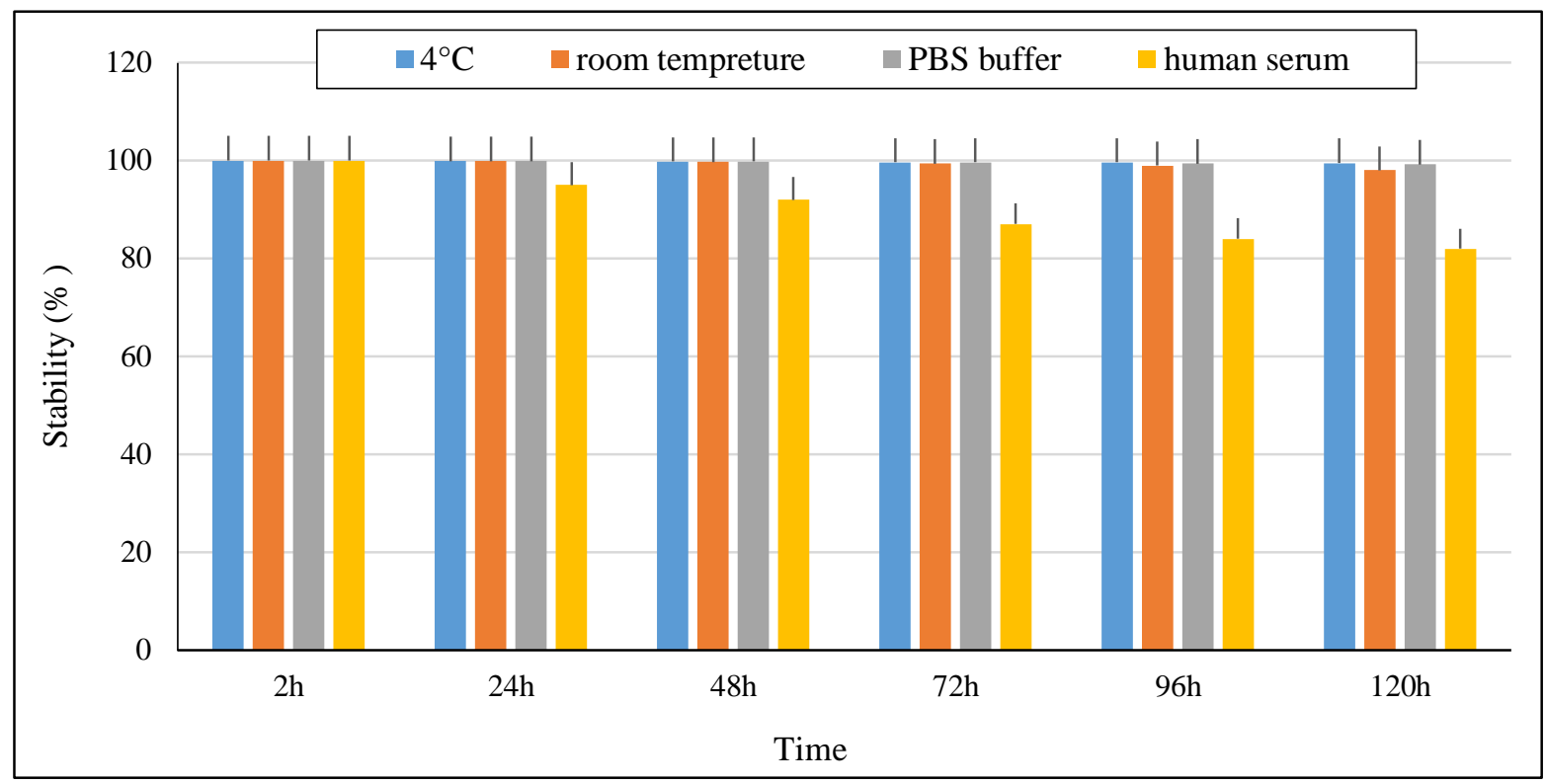

Figure 2. In vitro stability of radiolabeled avastin after storing at various conditions for different times.

\section{3-4- Integrity}

The samples were analyzed by SDS-PAGE. As shown in Figure 3 SDS-PAGE of labelled, conjugated, and native avastin showed no detectable modification of the molecule (Figure 4).

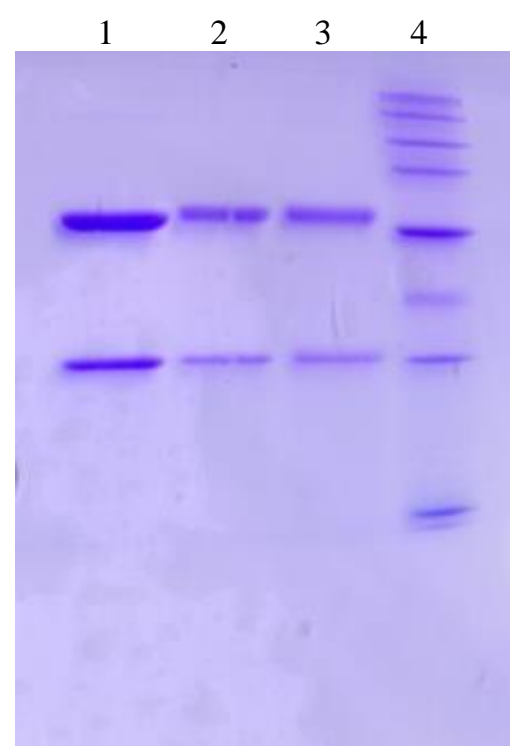

Figure 3. SDS-PAGE of ${ }^{153} \mathrm{Sm}$-DOTA- avastin performed in nonreducing condition showing Ladder (lane 4) native mAb (lane 3), conjugated (lane 2), and labelled (lane 1).

\section{3-4- Immunoreactivity}

Figure 4 shows the percentage of the ${ }^{153} \mathrm{Sm}$-DOTA- avastin mAb immonoreactivity compared with that of ${ }^{153} \mathrm{Sm}-$ DOTA-NHS only. An immonoreactivity was determined for the ${ }^{153} \mathrm{Sm}$-DOTA- avastin with $89 \pm 1.4 \%$ cell binding, in comparison to radiolabeled DOTA-NHS with $0.5 \%$ cell binding. These results show in vitro stability and specific immunoractivity against VEGF on the cells. 


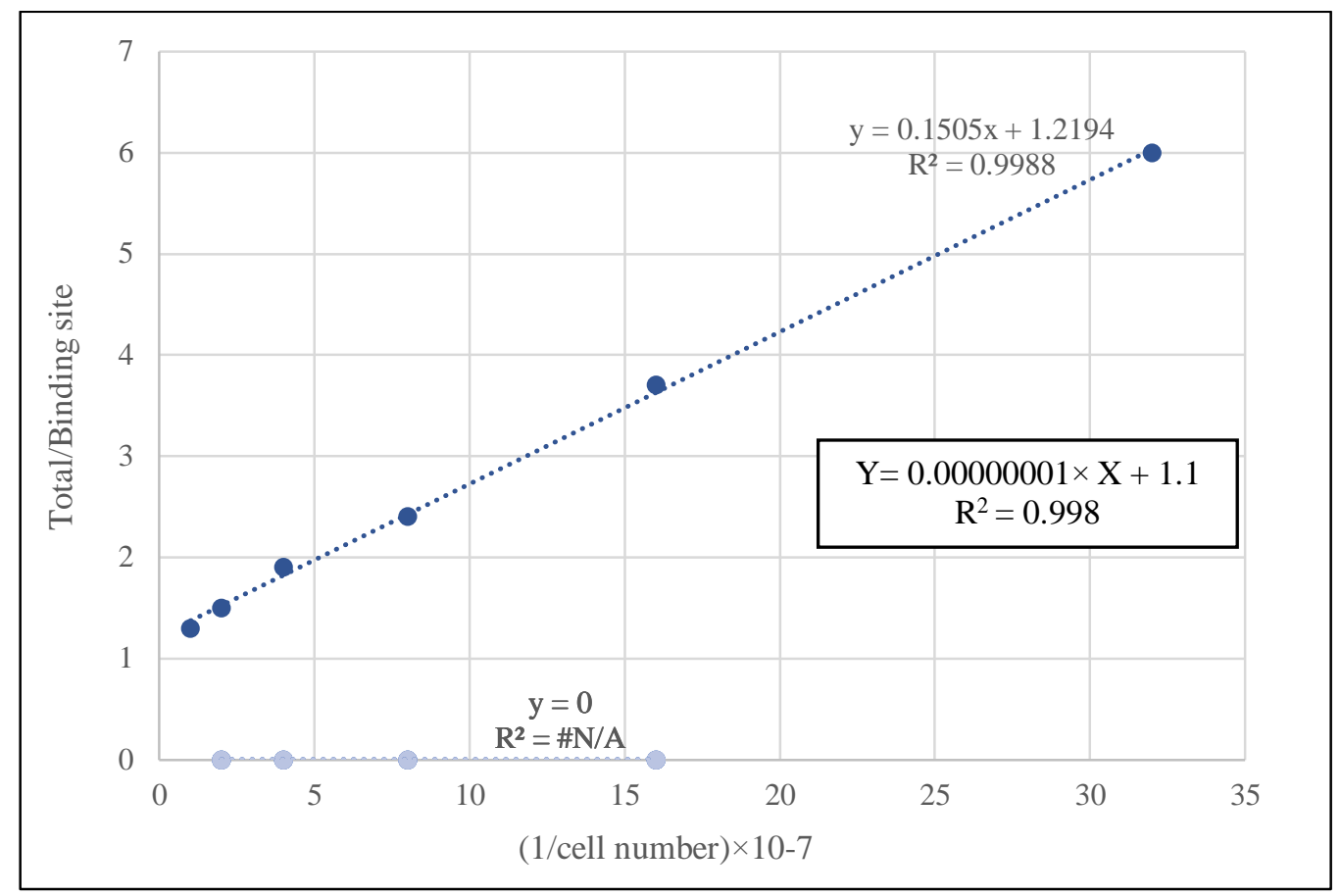

Figure 4. The immunoreactivity fraction shows the ability of the complex to bind to SW480 cells.

\section{3-5- Effect of ${ }^{153}$ Sm-DOTA-Avastin on Cell Proliferation}

The cytotoxicity of the complex on the SW480 cell line is shown in Figure 5A-C. Growth inhibition was time and dose dependent. The proliferation of cells treated with ${ }^{153} \mathrm{Sm}$-DOTA- avastin were inhibited notably compared with control cell groups, and the significant inhibition was $77 \pm 3 \%(\mathrm{p}<0.05)$ on the thirday. However, the cytotoxicity of ${ }^{153} \mathrm{Sm}$ (hot) and avastin alone at equal concentrations of the complex on SW480 cells were $27 \%$ and $4 \%$ respectively.

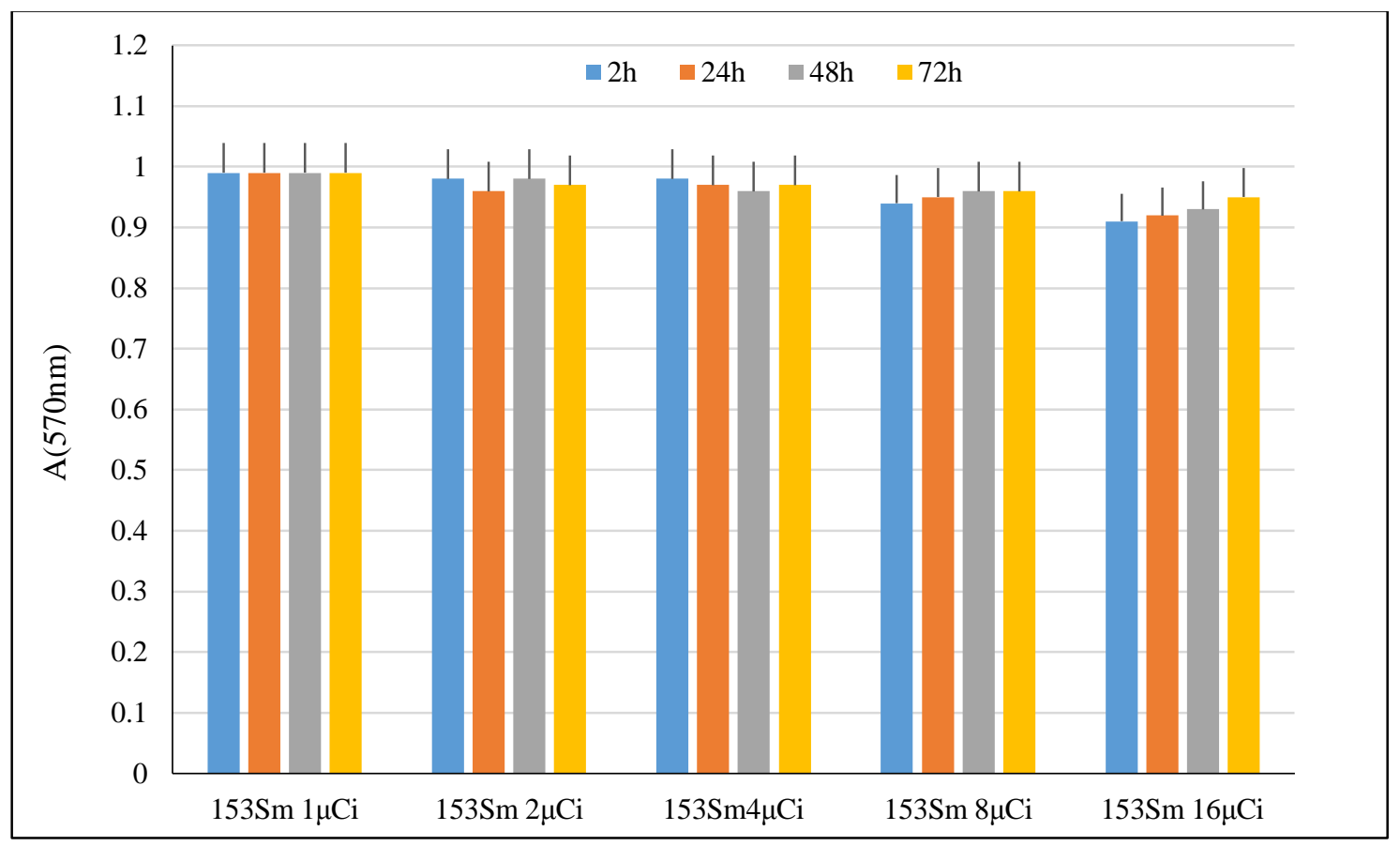

Figure 5A. Effect of ${ }^{153} \mathrm{SmCl3}$ on viability of SW480 cells. 


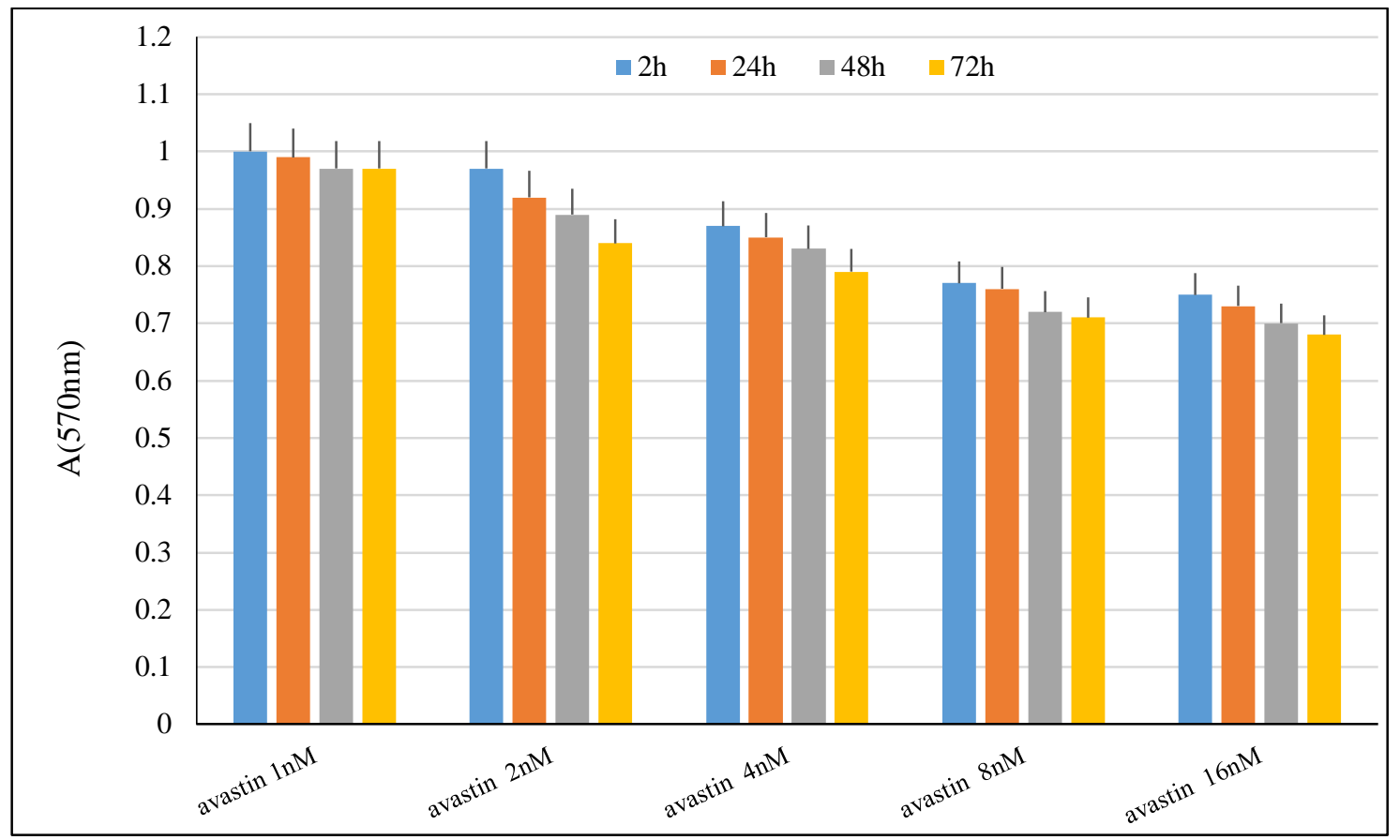

Figure 5B. Effect of avastin on viability of SW480 cells.

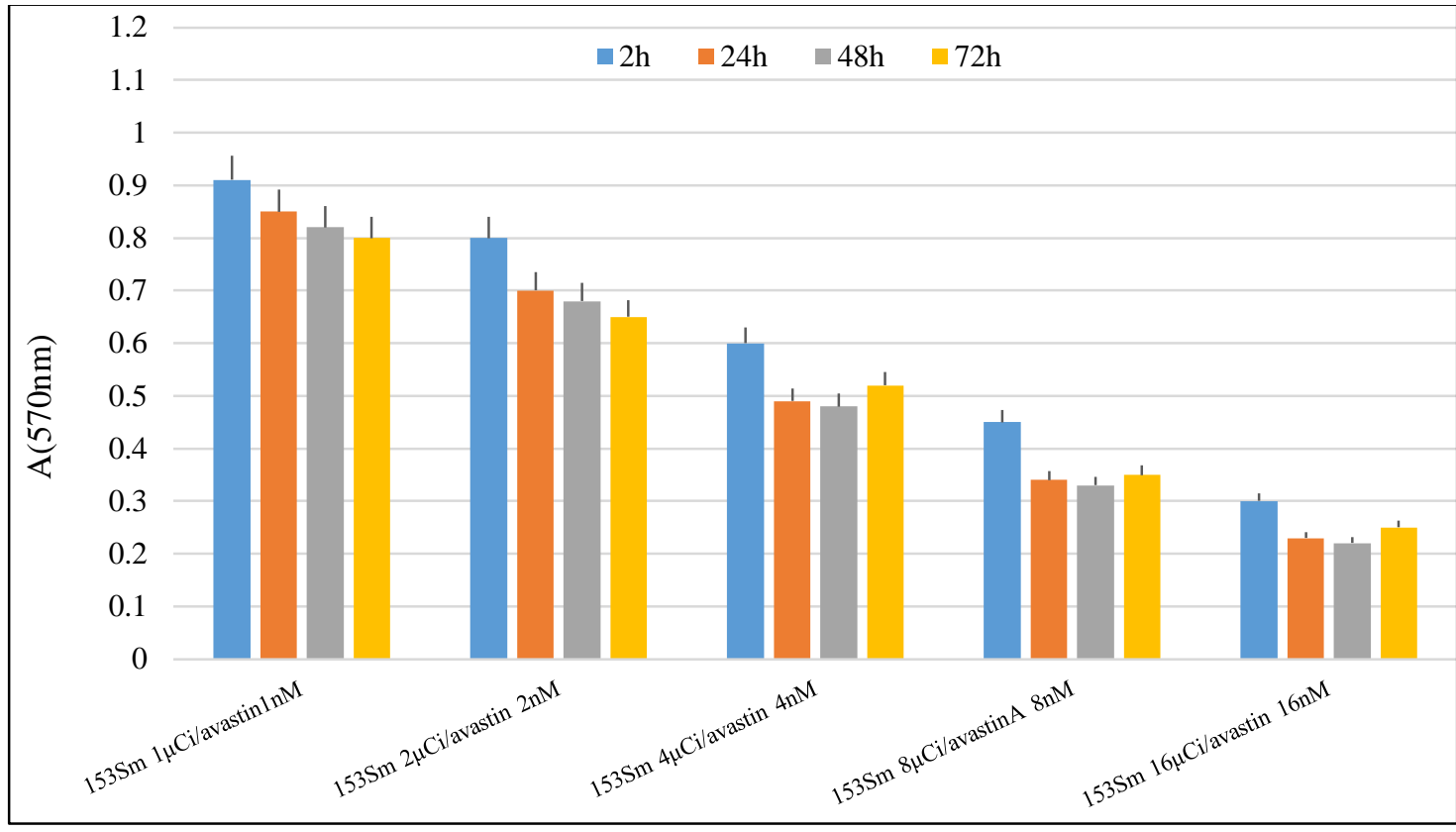

Figure 5C. Effect of ${ }^{153} \mathrm{Sm}$-DOTA- avastin (radioimmonutherapy) on viability of SW480 cells.

\section{3-6- Biodistribiotion Studies}

The distribution of ${ }^{153} \mathrm{SmCl} 3$ and ${ }^{153} \mathrm{Sm}$-DOTA-avastin was determined among the tissues for normal rats. Figure 6A shows the biological distribution of ${ }^{153} \mathrm{SmCl} 3$ in wild-type mice. The highest uptakes were observed in the bone and liver. The contrast of the liver, the high uptakes rate in the bone remains almost constant for 48 hours. Other organs did not significant uptake. In the same way, the distribution of ${ }^{153} \mathrm{Sm}$-DOTA-avastin in mice was tested. High uptake is observed in the lungs, spleen, heart and blood organs (Figure 6B). 


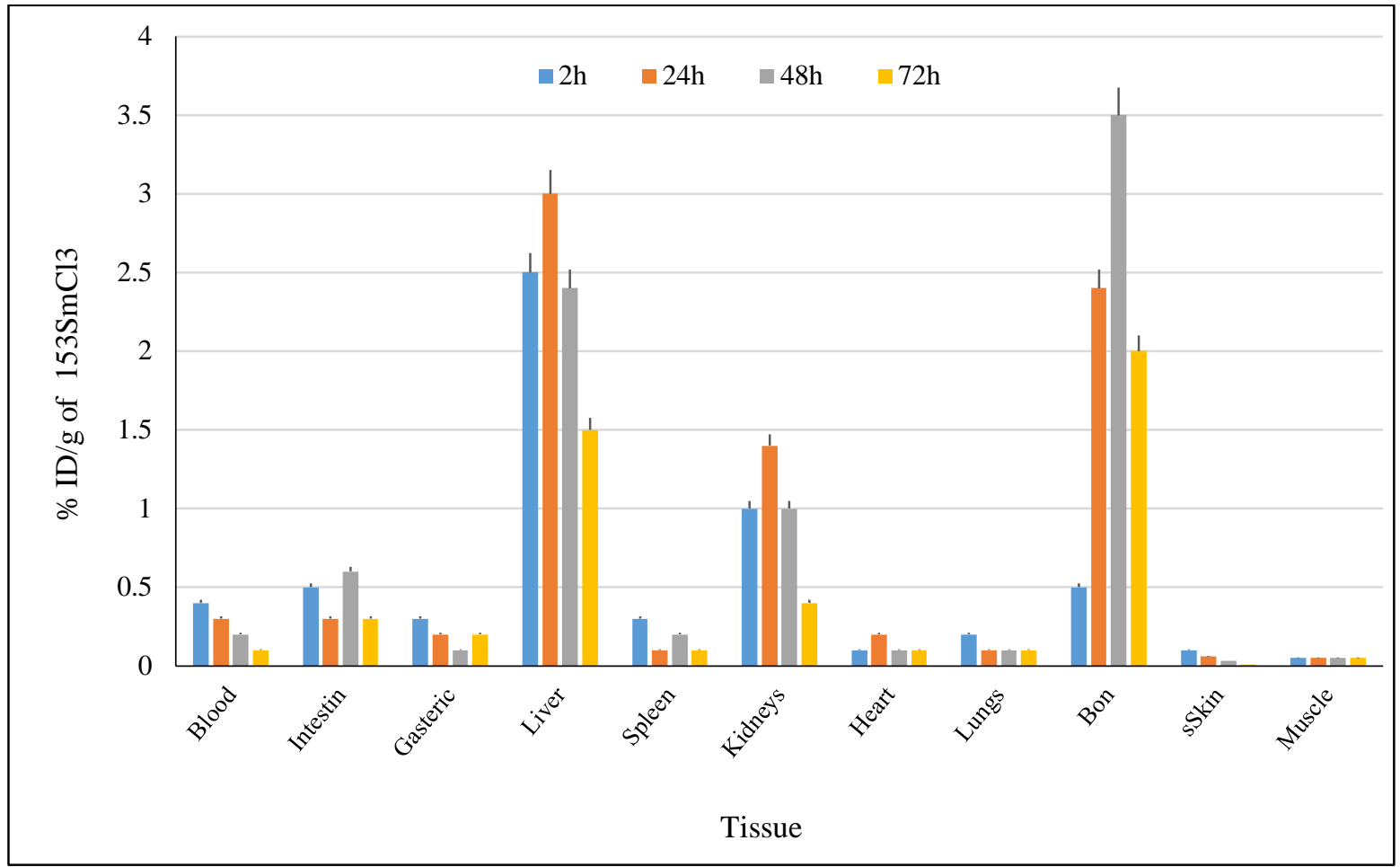

Figure 6A. Tissue distribution of ${ }^{153} \mathrm{Sm}$ activity in normal mice after injection of ${ }^{153} \mathrm{SmCl}_{3}$. Values are presented as percent injected dose per gram of tissue $(\% \mathrm{ID} / \mathrm{g}, \mathrm{n}=3)$

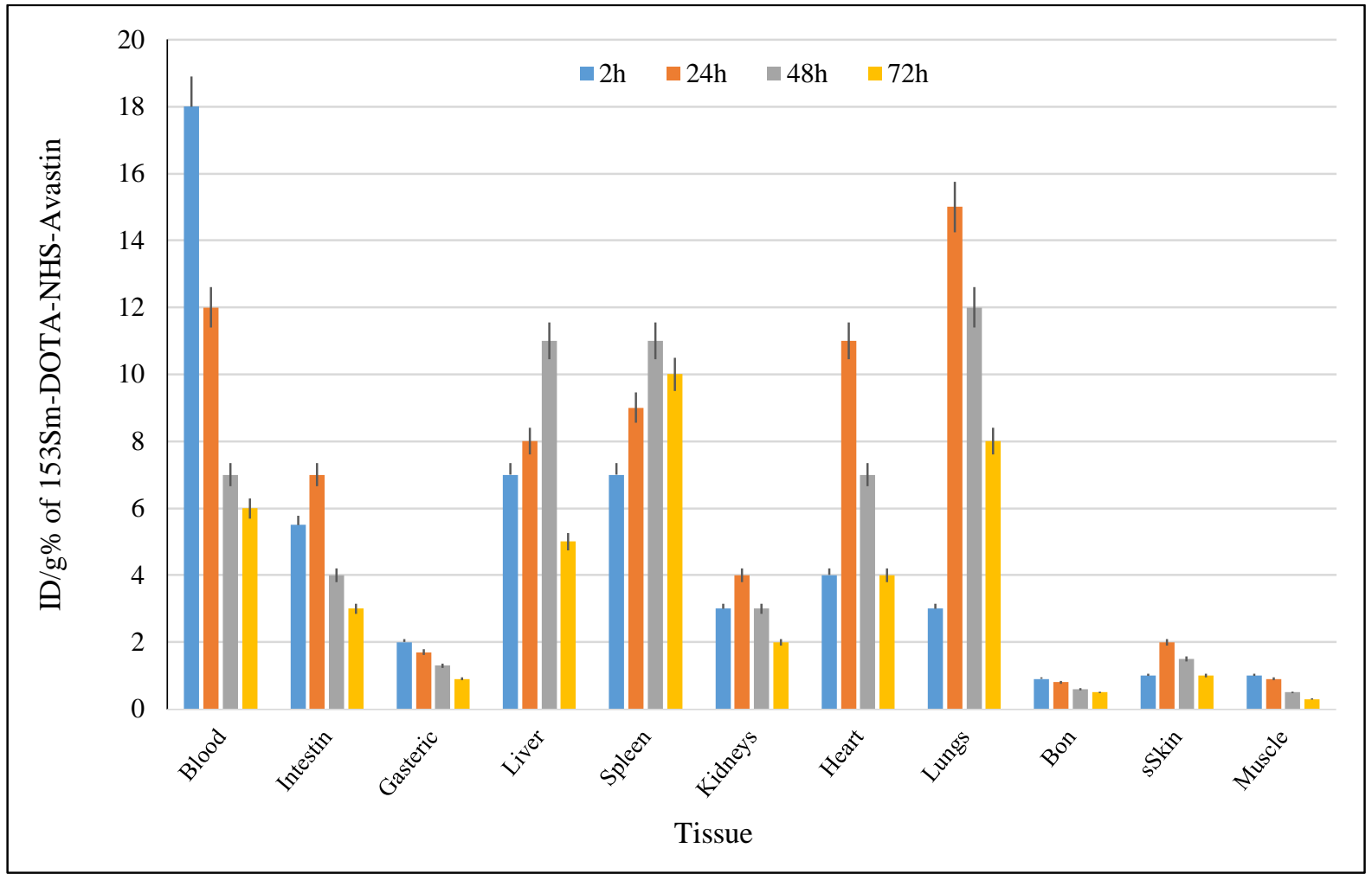

Figure 6B. Tissue distribution of ${ }^{153} \mathrm{Sm}$ activity in normal mice after injection of ${ }^{153} \mathrm{Sm}$-DOTA-avastin values are presented as percent injected dose per gram of tissue $(\% \mathrm{ID} / \mathrm{g}, \mathrm{n}=3)$.

Angiogenesis is a common feather of all metastatic tumors, and the development of antiangiogenic strategies aimed at halting cancer progression by inhibiting the growth of the new blood vessels is a major area of interest in clinical oncology [2-5].

Targeted and biological therapies have been investigated as the methods of improving anticancer therapy for many years. Such agents have several general characteristics that make them attractive options as anticancer drugs, but the most important one is the potential to improve the diagnostic outcomes with limited toxicity [18]. Several agents have 
been approved for the treatment of cancer as a result of this approach, the most notably of which are trastuzumab (Herceptin) for the treatment of human epidermal growth factor receptor-2 (HER2)-positive metastatic breast cancer and rituximab (MabThera) for the treatment of non-Hodgkin's lymphoma [19, 20]. One approach that was postulated as early as 1971 is the targeted therapy that can inhibit tumor angiogenesis, which has a critical role in the development of cancer [27]. The humanized monoclonal antibody bevacizumab, directed against vascular endothelial growth factor (VEGF), is the first and currently the only anti-angiogenic agent to be approved for cancer therapy [1]. Approval of bevacizumab was based on the results of several crucial phase II and III studies in patients with previously untreated metastatic CRC [6]. The antibody is currently scrutinized in at least 11 clinical case studies (Phase I-III) for various cancers as per information available from the dedicated avastin website. It has shown promise albeit to some extent in breast and small cell renal carcinoma but the outlook is hardly promising in prostate cancer [3-5, 10]. Bevacizumab is being used in combination with different chemotherapy drugs for the treatment of prostate cancer in various phases such as Phase II/Phase III of clinical case studies. It suggests that bevacizumab and chemotherapy in combination are possibly showing promise with respect to prostate cancer although the results of these trials are not expected in the near future. None of the experiments, however, involves the combination of bevacizumab with targeted radionuclide therapy.

\section{4- Conclusion}

In the present study we reported production a new radiopharmaceutical for specific targeted therapy. The ${ }^{153} \mathrm{SmCl}_{3}$ produced in the research reactor according to the regular methods. For labeling, firstly, avastin was purified, desalted and conjugated with DOTA-NHS. The chromatographic profile of the conjugated antibody was high and easy to accomplish through ascending paper chromatography (Whatman $\mathrm{n}^{\circ} 1$ ) and Instant Thin Layer Chromatographic (ITLCSG). To improve the labeling efficiency, a wide range of antibody/DOTA-NHS ratios were studied. According to Pippin et.al [15], the method to determine the number of chelating groups per mAb described produces the precise numbers of chelating that had really attached to the antibody molecule during this experiment were between the ratios of 1.1-1.7. This can explain excellent successful optimization conditions such as buffer type selection, antibody purification and other agents that effect on the conjugation. Also the conjugated antibody derived in the molar ratio of 1:20 presented higher radiochemical purity than those derived with higher molar ratios when radiolabel immediately after purification.

After conjugation, DOTA-avastin solutions were mixed with ${ }^{153} \mathrm{SmCl} 3$ solution and incubated in various buffers and at different temperatures to find the best time and condition scales for labeling. After an hour of radioconjugate incubation at $43^{\circ} \mathrm{C}$ in the bicarbonate buffer $(0.25 \mathrm{Mm}, \mathrm{pH} 9.2)$, total labeling and formulation of ${ }^{153} \mathrm{Sm}-\mathrm{DOTA}-$ avastin took about $1.5 \mathrm{~h}$ by means of PD10 columns with a yield of $95 \%$ and radiochemical purity of $99 \%$ in optimized condition.

The derived immunoconjugates stored for 4 day under different conditions showed good radiochemical purity results, which might indicate that no free radiometal was detected. These conditions were favorable for the derived immunoconjugates storage. Thus DOTA-avastin is capable of stably chelating ${ }^{153} \mathrm{Sm}$, which is mandatory for using

${ }^{153} \mathrm{Sm}$ radiolabelled avastin for therapy in patients with high expression of VEGF including all of the cancers.

The biodistribution of radiometal labelledbevacezumab has already been shown by ${ }^{89} \mathrm{Zr}$ or ${ }^{111}$ In [14]. In our study, the uptakes of the ${ }^{153} \mathrm{SmCl} 3$, morever, were observed in the bone and liver for its delivery and the possible route of accumulation and high penetration efficiency respectively. Also the liver uptake of the cation is comparable to many other radio-metals mimicking ferric cation accumulation. The Lung, muscle and skin do not demonstrate a significant uptake that is in the accordance with other cations accumulation. In contrast, high uptake of ${ }^{153}$ Sm-DOTA- avsatin was observed in blood, the spleen, lungs and heart, especially at 24 and $48 \mathrm{~h}$ time-points. These organs normally have very good tissue perfusion and a significant blood volume. By taking into consideration the high levels of radiotracer measured in blood we can infer that blood pooling effects are responsible for the levels of radioactivity measured in these tissues. Conversely, the reduced tissue blood volume may explain the low uptake of radiotracer into the bone. Results indicated that ${ }^{153} \mathrm{Sm}$-DOTA-avastin is more stable in vivo.

\section{5- Ethical Approval}

All procedures performed in studies involving human participants were in accordance with the ethical standards of the institutional and/or national research committee and with the 1964 Helsinki declaration and its later amendments or comparable ethical standards.

\section{6- References}

[1] Dvorak, Harold F., Lawrence F. Brown, Michael Detmar, and Ann M. Dvorak. "Vascular permeability factor/vascular endothelial growth factor, microvascular hyperpermeability, and angiogenesis." The American journal of pathology 146, no. 5 (1995): 10291039.

[2] Shibuya, Masabumi. "Structure and Function of VEGF/VEGF-Receptor System Involved in Angiogenesis." Cell Structure and Function 26, no. 1 (2001): 25-35. doi:10.1247/csf.26.25. 
[3] Breier, Georg. "Functions of the VEGF/VEGF Receptor System in the Vascular System." Seminars in Thrombosis and Hemostasis 26, no. 05 (2000): 553-560. doi:10.1055/s-2000-13212.

[4] Carmeliet, Peter. "VEGF as a Key Mediator of Angiogenesis in Cancer." Oncology 69, no. 3 (2005): 4-10. doi:10.1159/000088478.

[5] Rüegg, Curzio, Meriem Hasmim, Ferdy J. Lejeune, and Gian Carlo Alghisi. "Antiangiogenic Peptides and Proteins: From Experimental Tools to Clinical Drugs.” Biochimica et Biophysica Acta (BBA) - Reviews on Cancer 1765, no. 2 (April 2006): 155-177. doi:10.1016/j.bbcan.2005.09.003.

[6] Mitra, Amitava, Anjan Nan, John C. Papadimitriou, Hamidreza Ghandehari, and Bruce R. Line. "Polymer-Peptide Conjugates for Angiogenesis Targeted Tumor Radiotherapy." Nuclear Medicine and Biology 33, no. 1 (January 2006): 43-52. doi:10.1016/j.nucmedbio.2005.09.005.

[7] Garcia-Barros, M. “Tumor Response to Radiotherapy Regulated by Endothelial Cell Apoptosis.” Science 300, no. 5622 (May 16, 2003): 1155-1159. doi:10.1126/science.1082504.

[8] Hsu, Jerry Y., and Heather A. Wakelee. "Monoclonal Antibodies Targeting Vascular Endothelial Growth Factor." BioDrugs 23, no. 5 (October 2009): 289-304. doi:10.2165/11317600-000000000-00000.

[9] Grothey, Axel, and Lee M. Ellis. "Targeting Angiogenesis Driven by Vascular Endothelial Growth Factors Using Antibody-Based Therapies.” The Cancer Journal 14, no. 3 (May 2008): 170-177. doi:10.1097/ppo.0b013e318178d9de.

[10] Karagiannis, Tom C. "Comparison of different classes of radionuclides for potential use in radioimmunotherapy." Hell J Nucl Med 10, no. 2 (2007): 82-8.

[11] Khorami-Moghadam, Alireza, Amir Jalilian, Kamal Yavari, Bahram Bolouri, Ali Bahrami-Samani, and Mohammad GhannadiMaragheh. "Production and quality control of [166Ho]-DOTA-bevacizumab for therapeutic applications." Journal of Radioanalytical and Nuclear Chemistry 292, no. 3 (2012): 1065-1073.

[12] Xiao, Jie, Xiaobo Xu, Xiao Li, Yanli Li, Guobing Liu, Hui Tan, Hua Shen, Hongcheng Shi, and Dengfeng Cheng. "Re-188 Enhances the Inhibitory Effect of Bevacizumab in Non-Small-Cell Lung Cancer." Molecules 21, no. 10 (September 30, 2016): 1308. doi:10.3390/molecules21101308.

[13] Fani, M, S Vranjes, S.C Archimandritis, S Potamianos, S Xanthopoulos, P Bouziotis, and A.D Varvarigou. "Labeling of Monoclonal Antibodies with 153Sm for Potential Use in Radioimmunotherapy.” Applied Radiation and Isotopes 57, no. 5 (November 2002): 665-674. doi:10.1016/s0969-8043(02)00181-1.

[14] Fani, Melpomeni, Stavros Xanthopoulos, Spyridon C. Archimandritis, Nikolaos Stratis, Penelope Bouziotis, George Loudos, and Alexandra D. Varvarigou. "Biodistribution and scintigraphic studies of 153Sm-labeled anti-CEA monoclonal antibody for radioimmunoscintigraphy and radioimmunotherapy." Anticancer research 23, no. 3A (2003): 2195-2199.

[15] Kraeber-Bodéré, Françoise, Anil Mishra, Philippe Thédrez, Alain Faivre-Chauvet, Manuel Bardiès, S. Imai, Jean Le Boterff, and Jean-François Chatal. "Pharmacokinetics and Biodistribution of Samarium-153-Labelled OC125 Antibody Coupled to CITCDTPA in a Xenograft Model of Ovarian Cancer.” European Journal of Nuclear Medicine 23, no. 5 (May 1996): $560-567$. doi:10.1007/bf00833392.

[16] Izard, M. E., G. R. Boniface, K. L. Hardiman, M. W. Brechbiel, O. A. Gansow, and K. Z. Walker. “An Improved Method for Labeling Monoclonal Antibodies with Samarium-153: Use of the Bifunctional Chelate 2-(p-Isothiocyanatobenzyl)-6Methyldiethylenetriaminepentaacetic Acid.” Bioconjugate Chemistry 3, no. 4 (July 1992): 346-350. doi:10.1021/bc00016a015.

[17] Pippin, C. Greg, Tammy A. Parker, Thomas J. McMurry, and Martin W. Brechbiel. "Spectrophotometric Method for the Determination of a Bifunctional DTPA Ligand in DTPA-Monoclonal Antibody Conjugates." Bioconjugate Chemistry 3, no. 4 (July 1992): 342-345. doi:10.1021/bc00016a014.

[18] Adler, Mark J., and Dimiter S. Dimitrov. "Therapeutic Antibodies Against Cancer.” Hematology/Oncology Clinics of North America 26, no. 3 (June 2012): 447-481. doi:10.1016/j.hoc.2012.02.013.

[19] Garnock-Jones, Karly P., Gillian M. Keating, and Lesley J. Scott. “Trastuzumab.” Drugs 70, no. 2 (January 2010): 215-239. doi:10.2165/11203700-000000000-00000.

[20] Dotan, Efrat, Charu Aggarwal, and Mitchell R. Smith. "Impact of rituximab (Rituxan) on the treatment of B-cell non-Hodgkin's lymphoma." Pharmacy and Therapeutics 35, no. 3 (2010): 148.

[21] Arjaans, Marlous, Carolina P. Schröder, Sjoukje F. Oosting, Urania Dafni, Josée E. Kleibeuker, and Elisabeth G.E. de Vries. "VEGF Pathway Targeting Agents, Vessel Normalization and Tumor Drug Uptake: From Bench to Bedside." Oncotarget 7, no. 16 (January 14, 2016). doi:10.18632/oncotarget.6918.

[22] Aaron P. B.,Clinical biomarkers of angiogenesis inhibition. Cancer Metastasis Review, vol.27, no.3, (2008), p. $415-434$.

[23] Zhao, Y., and A. A. Adjei. "Targeting Angiogenesis in Cancer Therapy: Moving Beyond Vascular Endothelial Growth Factor." The Oncologist 20, no. 6 (May 22, 2015): 660-673. doi:10.1634/theoncologist.2014-0465. 
[24] Chong, G., and N.C. Tebbutt. "Using Bevacizumab with Different Chemotherapeutic Regimens in Metastatic Colorectal Cancer: Balancing Utility with Low Toxicity.” Therapeutic Advances in Medical Oncology 2, no. 5 (July 14, 2010): $309-317$. doi:10.1177/1758834010375096.

[25] Strickler, J. H., and H. I. Hurwitz. "Bevacizumab-Based Therapies in the First-Line Treatment of Metastatic Colorectal Cancer." The Oncologist 17, no. 4 (April 1, 2012): 513-524. doi:10.1634/theoncologist.2012-0003.

[26] De Gramont, Aimery, and Eric Van Cutsem. "Investigating the Potential of Bevacizumab in Other Indications: Metastatic Renal Cell, Non-Small Cell Lung, Pancreatic and Breast Cancer.” Oncology 69, no. 3 (2005): 46-56. doi:10.1159/000088483.

[24] Yavari, Kamal. "Production and Quality Control of 153Sm-DOTA-Avastin." Journal of Ardabil University of Medical Sciences 11, no. 4 (2011): 380-389.

[27] Nagengast, W. B., E. G. de Vries, G. A. Hospers, N. H. Mulder, J. R. de Jong, H. Hollema, A. H. Brouwers, G. A. van Dongen, L. R. Perk, and M. N. Lub-de Hooge. "In Vivo VEGF Imaging with Radiolabeled Bevacizumab in a Human Ovarian Tumor Xenograft.” Journal of Nuclear Medicine 48, no. 8 (August 1, 2007): 1313-1319. doi:10.2967/jnumed.107.041301. 